\title{
On the Role of Job Assignment in a Comparison of Education Systems
}

\section{July 06, 2009}

Katsuya Takii, Osaka University

Ryuichi Tanaka, Tokyo Institute of Technology

\section{【キーワード】Job assignment, Human capital, Education system, D31 D72 H42 I22 011015}

【要約】This paper reexamines how differences in systems for financing education influence GDP by highlighting a neglected function of education policy: it affects the magnitude of gains from job assignment. When more productive jobs demand more skill, privately financed education can increase productivity gains from matching between jobs and skill by increasing the availability of highly educated people. This differs from the standard argument that publicly financed education increases the total amount of human capital by equalizing educational opportunities. It is shown that if job opportunities have large variations in productivity, education policy may face a serious efficiency--equity trade-off.

We are grateful to the seminar participants at the Osaka Workshop on Economics of Institutions and Organizations and Tohoku University for helpful comments. Financial support from Seimeikai and the Japanese Ministry of Education, Culture, Sports, Science and Technology (Grant-in-Aid for Scientific Research) is gratefully acknowledged. Katsuya Takii: Osaka School of International Public Policy, Osaka University, 1-31, Machikaneyama, Toyonaka, Osaka, 560-0043, Japan. (takii@osipp.osaka-u.ac.jp) Ryuichi Tanaka: Graduate School of Information Science and Engineering, Tokyo Institute of Technology, 2-12-1 O-okayama, Meguro-ku, Tokyo 152-8552, Japan. (tanaka@mei.titech.ac.jp) 


\section{Introduction}

A publicly financed education system redistributes educational resources from rich to poor households through taxes and aims to provide equal opportunity for children to receive schooling regardless of their family background. One of the main concerns is whether or not this egalitarian education policy causes a serious trade-off between efficiency and equity. The literature on the economics of education points out several mechanisms through which the public education system increases or decreases gross domestic product (GDP), and provides useful insights for understanding when and how the trade-off emerges.

On the one hand, it is common in the literature to assume declining marginal returns to educational investment, which favors a publicly financed system (e.g., Loury 1981, Glomm and Ravikumar 1992, Bénabou 1996, 2002, Fernández and Rogerson 1998, and Takii and Tanaka 2009). ${ }^{1} \quad$ If the marginal returns from investment in human capital decline as agents spend more resources on education, the public system can increase the total amount of human capital in an economy, and therefore GDP, by equalizing educational opportunities because the poor's marginal returns to investment are higher than those of the rich. ${ }^{2}$

On the other hand, Glomm and Ravikumar (1992) and Takii and Tanaka (2009) point out that there are disadvantages of a publicly financed education system in

\footnotetext{
${ }^{1}$ To give a public education system the role of equalizing educational opportunities, it is also common to assume there is a liquidity constraint or that parents have warm-glow altruism and paternalistic altruism so that education can be treated as a consumption good as well as an investment good.

${ }^{2}$ This mechanism is fairly strong. Takii and Tanaka (2009) show that when the intergenerational income elasticity is less than one, a public system with equal provision of resources to students yields higher GDP than a private system, even if the aggregate production function is convex in individual skill.
} 
comparison with a privately financed education system. Glomm and Ravikumar (1992) show that the private system can generate higher GDP than the public system if a child can choose to exert effort in the accumulation of human capital. Takii and Tanaka (2009) find that if private schools are better able to screen students based on their ability than public schools, there is a production structure in which the private system attains higher GDP than the public system.

The purpose of this paper is to contribute to these discussions by highlighting an important function of education policy neglected in the literature: it affects the distribution of educated people and thus the magnitude of gains from job assignment in the labor market. Without exception, the existing literature assumes that there is an aggregated measure of human capital that summarizes all the impacts of individual human capital accumulation on GDP. We argue that this assumption masks how an education policy influences the gains from assignment.

To highlight the novel role of an education policy, we incorporate a standard positive assortative assignment model based on Sattinger (1979) into an overlappinggenerations model with human capital accumulation through tax-financed public schooling or tuition-financed private schooling, abstracting away the effects of effort level and ability tracking on the accumulation of human capital. Under this setup, the public system always yields higher GDP than a private education system if there is no assignment problem in the labor market. It is shown that a private system can attain higher GDP because it increases productivity gains from assignment by increasing the availability of highly educated people.

We emphasize the importance of three distinctive assumptions behind the positive assortative assignment model, which are essential to understanding this result. The first assumption is that the quality and the quantity of workers are not perfect substitutes. ${ }^{3}$ When the quality of workers can be substituted by the quantity of workers,

\footnotetext{
${ }^{3}$ Sattinger (1980) discusses convincingly the importance of imperfect substitution between the
} 
the returns from employing doubly productive workers are equivalent to those from doubling the number of workers regardless of the structure of the industry and firm. In this case, the total productivity of the economy is influenced only by the total amount of human capital. However, when the quantity and quality of workers are not perfect substitutes, a firm may seek a highly talented expert rather than a large number of ordinary workers. If there is no expert in an economy, the firm will incur a huge loss. An education policy changes the availability of qualified workers, so it can influence not only the total productive capacity of workers but also the pattern of assignment between skill and productive job opportunities.

The second assumption is that job opportunities are heterogeneous. ${ }^{4}$ Without heterogeneity among jobs, there are no gains from assignment that may potentially provide advantages to a private education system. Among the papers on the comparison between public and private education systems, Bénabou (1996) and Takii and Tanaka (2009) analyze models in which the quality and quantity of human capital are not perfect substitutes. However, as they do not have any variation in jobs, their measures of aggregate human capital summarize all the impacts of individual human capital accumulation on GDP.

The third assumption is that jobs that are more productive demand more skill. That is, job-specific productivity is complementary to skill. With this complemenquality and quantity of workers for assignment. The importance of the imperfect substitution between skilled workers and unskilled workers can also be understood from the arguments regarding skill-biased technological change (e.g., Katz and Murphy 1992).

${ }^{4}$ Considerable evidence suggests that job opportunities are heterogeneous. For example, the literature finds that there are substantial and persistent differences in productivity between plants and between firms (e.g., Baily, Hulten and Campbell 1992). Researchers also find that the observed and unobserved characteristics of a firm influence wage payments after controlling for the individual effect using matched employer-employee data (e.g., Abowd, Kramarz and Margolis 1999). 
tarity, it is well known that talented workers are assigned to productive jobs. ${ }^{5}$ Hence, the existence of a few highly skilled workers causes disproportional impacts on aggregate productivity compared with that of ordinary workers because they are not only productive, but can also utilize their skill to succeed in highly productive jobs. Hence, the aggregate productivity might be larger by educating a few highly skilled workers rather than a large number of reasonably trained workers. This mechanism provides a novel channel, through which the private system increases GDP.

This paper shows that, in the short run, an increase in the variations in productivity across jobs (in the sense of mean preserving spread) increases the productivity gains from a private education system more than those from a public education system. Because of the complementarity between the productivity of jobs and skill, the existence of highly productive job opportunities significantly increases the marginal

\footnotetext{
${ }^{5}$ There is plenty of suggestive evidence showing that more productive jobs demand more skill and that there is positive assortative matching in the labor market. For example, evidence shows that new technologies are skill biased, which means that advanced technologies demand more skill (e.g., Krusell et al. 2000). Other factors also demand high levels of skill. Chandler (1977) demonstrates that the modern organizational structure demands skilled workers to process information. Bresnahan, Brynjolfsson and Hitt (2002) also show that reorganization accommodated by IT investment demands more skilled workers. More directly, using a model similar to this paper, Takii (2008) shows that the assumption of complementarity between firm-specific productivity and skill is consistent with observed persistent productivity differences across firms. Recently, researchers have tried to find more direct evidence using matched employer-employee data. Although controversial evidence is found by some researchers (e.g., Abowd, Kramarz and Margolis 1999), other researchers criticize the identification strategy used by these authors (e.g., Eeckhout and Kircher (2009)) and develop more sophisticated methods to examine empirically the positive assortative matching hypothesis in a labor market. Among them, Mendes, Van den Berg and Lindeboom (2007) and Lopes de Melo (2008) provide evidence based on their methods that supports a positive assortative matching hypothesis. At least, there is no evidence against a positive assortative matching hypothesis if observable characteristics are not controlled for, which is more relevant in this paper.
} 
product of skill. Hence, an increase in the availability of highly skilled workers improves aggregate productivity. As the private education system can produce more highly qualified workers than the public system, an increase in the variations in productivity increases the relative advantage of private education.

This result may not be true in the long run. As the large productivity differences bring better opportunities for advantaged people, it increases income inequality in the long run, which may make income transfer by a public education system more attractive. Nonetheless, it is shown that the private system generates higher long-run GDP than the public system does if jobs have sufficient variations in productivity. Hence, if jobs have large variations in their productivity, a serious efficiency-equity trade-off may occur even in the long run.

As well as contributing to the literature on the comparison of education systems, this paper also contributes to the literature of positive assortative assignment models. Positive assortative assignment models have a long history (e.g., Becker 1973, Sattinger 1979, and Kremer 1993 ). However, these papers typically assume the existence of exogenous distributions of assigned variables. More recently, researchers have started to endogenize the distribution (e.g., Acemoglu 1997, Jovanovic 1998, 2009 and Takii 2008). In particular, similarly to this paper, Acemoglu (1997) and Jovanovic $(1998,2009)$ endogenize the distribution of skill. However, no paper analyzes how an education policy influences the gains from assignment.

The rest of this paper is organized as follows. Section 2 describes the model. Sections 3 and 4 compare the two education systems in the short run and in the long run, respectively. Section 5 discusses some extensions and concludes. 


\section{The Model}

In this section, we incorporate positive assortative job assignment between jobspecific productivity and the human capital of workers into a standard overlappinggenerations model with an educational choice. We first describe the production side and solve the static equilibrium, and then model the investment in human capital.

Static Equilibrium: Our static assignment model is a standard one, which was originally analyzed by Sattinger (1979). The labor market of the economy is represented by a continuum of workers and jobs in each period. Although this paper calls a unit of production "a job", a job can alternatively be interpreted as a firm with one unit mass of workers.

The populations of both jobs and workers are normalized to one. Each job consists of job-specific productivity ${ }^{6}, z$, the logarithm of which is normally distributed with mean $\mu_{z}$ and variance $\sigma_{z}^{2}: \ln z \sim N\left(\mu_{z}, \sigma_{z}^{2}\right)$. The distribution of $z$ is assumed to be constant over time. We interpret a large $z$ as a result of large fixed specific resources that workers can use to make the job productive. Hence, the variance of $\ln z, \sigma_{z}^{2}$, can be interpreted as the variability of job opportunities.

Each worker is characterized by his/her human capital, $h_{t}$, which is also lognormally distributed: $\ln h_{t} \sim N\left(\mu_{h, t}, \sigma_{h, t}^{2}\right)$. Note that the distribution of $\ln h_{t}$ is a function of time, $t$. We endogenize this distribution later.

Assume that a job with productivity $z$ employs one worker with human capital $h_{t}$, and produces $y(z)$ by a production function, $y(z)=z h_{t}^{v}$, where $v>0$. This production function exhibits the complementarity between $z$ and $h_{t}$. For a simple

\footnotetext{
${ }^{6}$ Sattinger $(1979,1980)$ interprets his model as positive assortative matching between the skill of workers and a firm's real physical capital.
} 
explanation, we assume that jobs are perfect substitutes in a firm. In this case, the firm's profit maximization problem is equivalent to the job's profit maximization problem. As discussed later, this assumption can be relaxed without any changes in our result. The job chooses the human capital of a worker to maximize the profit:

$$
\chi_{t}(z)=\arg \max _{h_{t}}\left\{z h_{t}^{v}-w_{t}\left(h_{t}\right)\right\}
$$

where $w_{t}(h)$ is the wage payment for the workers with $h$ at date $t$. Note that the job determines the quality of workers but not the quantity. This means that the quantity of workers cannot be substituted for the quality. This is the assumption that deviates from standard human capital theory.

As the production function exhibits complementarity between job opportunities and skill, it is well known that job matching is positively assortative (e.g., Becker 1973). We characterize a positive assortative matching equilibrium. Let $\Phi(\bullet)$ denote a standard normal distribution. Because $\ln z \sim N\left(\mu_{z}, \sigma_{z}^{2}\right)$ and $\ln h_{t} \sim N\left(\mu_{h, t}, \sigma_{h, t}^{2}\right)$, a positive assortative equilibrium implies that:

$$
1-\Phi\left(\frac{\ln z-\mu_{z}}{\sigma_{z}}\right)=1-\Phi\left(\frac{\ln \chi_{t}(z)-\mu_{h, t}}{\sigma_{h, t}}\right), \text { for all } z \geq 0 .
$$

This equation means that a job in the top $x$ percentile of $z$ must select a worker in the top $x$ percentile of $h$. For the sake of simplicity, we assume that jobs and workers have a reservation wage and profit of zero, respectively. As the number of jobs is equal to the number of workers, nobody chooses the outside option and every agent can find a partner. Hence, a static equilibrium is fully characterized by $\chi_{t}(\cdot)$ and $w_{t}(\cdot)$ satisfying equations $(1)$ and $(2)$.

Because the function $\chi_{t}(\cdot)$ must satisfy the equation (2),

$$
\ln \chi_{t}^{-1}\left(h_{t}\right)=\frac{\sigma_{z}\left(\ln h_{t}-\mu_{h, t}\right)}{\sigma_{h, t}}+\mu_{z} .
$$

Consider a job with $\hat{z}=\chi_{t}^{-1}(\hat{h})$. This job requires a worker with $\hat{h}$ by definition of $\chi_{t}(\cdot)$. This means that if the maximization is characterized by the first- order 
condition, the following condition must be satisfied in the equilibrium:

$$
w_{t}^{\prime}(\hat{h})=v \chi_{t}^{-1}(\hat{h})(\hat{h})^{v-1}=\frac{v \check{y}_{t}(\hat{h})}{\hat{h}},
$$

where $\check{y}_{t}(\hat{h})=y\left(\chi_{t}^{-1}(\hat{h})\right)=\exp \left\{\mu_{z}+v \mu_{h, t}+\left(\frac{\sigma_{z}+v \sigma_{h, t}}{\sigma_{h, t}}\right)\left(\ln \hat{h}-\mu_{h, t}\right)\right\}$. Because the reservation wages of all workers are equal to zero and the choice of $\hat{h}$ is arbitrary, we can solve the wage function by integrating $w_{t}^{\prime}(h)$ with respect to $h$ :

$$
w_{t}(\hat{h})=\int_{0}^{\hat{h}} w_{t}^{\prime}(s) d s=L S_{t} \check{y}_{t}(\hat{h}),
$$

where $L S_{t}=\frac{v \sigma_{h, t}}{\sigma_{z}+v \sigma_{h, t}}$ is the labor share in production. As this wage function guarantees that the problem (1) is a convex problem for the job with $\hat{z}=\chi_{t}^{-1}(\hat{h})$, the first-order condition fully characterizes the job's profit maximization problem. Hence the derived $\chi_{t}(\cdot)$ and $w_{t}(\cdot)$ satisfy the static equilibrium conditions (1) and (2), which proves the existence of the equilibrium.

We can derive the GDP of the economy, $Y_{t}$, as follows:

$$
Y_{t}=E\left[z h_{t}^{v}\right]=E(z) E\left(h_{t}^{v}\right) A S_{t},
$$

where $E(z)=\exp \left(\mu_{z}+\frac{\sigma_{z}^{2}}{2}\right), E\left(h_{t}^{v}\right)=\exp \left(v \mu_{h, t}+\frac{v^{2} \sigma_{h, t}^{2}}{2}\right)$ and $A S_{t}=\exp \left[v \sigma_{z} \sigma_{h, t}\right]$. Note that GDP can be decomposed into three components: average productivity, $E(z)$, aggregate human capital $E\left(h_{t}^{v}\right)$ and the productivity gains from assignment, $A S_{t}$. Note that an increase in $\sigma_{h, t}$ increases $A S_{t}$. Because job-specific productivity and the skill of workers are complementary to each other, the matching of highly productive jobs and exceptionally high skill creates disproportional impacts on productivity. As an increase in $\sigma_{h, t}$ means that more extremely talented workers are available, it increases the productivity gains from assignment. This is the mechanism by which a private education system can increase aggregate productivity.

Note that if $\sigma_{z}=0, Y=E(z) E\left(H_{t}\right)$ and $w\left(h_{t}\right)=E(z) H_{t}$, where $H_{t}=h_{t}^{v}$. Hence, the result is equivalent to that of a linear production function with perfectly 
substitutable human capital $H_{t}$. This is the standard production function considered in previous studies (e.g., Glomm and Ravikumar 1992). It means that, without the heterogeneity of jobs, there are no gains from assignment and aggregate human capital can summarize all the impacts of human capital accumulation on GDP. In the following section, we take $\sigma_{z}=0$ as the benchmark case and investigate how the results change when $\sigma_{z}>0$.

Households: There is a continuum of overlapping-generation families $i$, the total mass of which is also normalized to unity. In each period, each family consists of one child, one young adult and one aged person. Each individual lives for three periods. In the first period, children obtain an education while they are still supported by their parents. In the second period, young individuals work, spend part of their income on current consumption and the education of their children, and save the rest. In the third period, old individuals consume their savings.

Human capital is formed according to individual ability $\xi_{t+1}^{i}$ (i.i.d. with mean 1), public education expenditure per pupil $g_{t}$ and private education expenditure per pupil $e_{t}^{i}$. Public education is financed by the proportional wage income tax: $g_{t}=$ $\tau_{t} \int w\left(h_{t}^{i}\right)_{t} d i$ where $\tau_{t} \in[0,1]$ is the tax rate. The production function of human capital is given by:

$$
h_{t+1}^{i}=\xi_{t+1}^{i}\left[\left(e_{t}^{i}\right)^{\theta}\left(g_{t}\right)^{1-\theta}\right]^{\alpha}, \theta \in\{0,1\}, \alpha \in(0,1)
$$

where $\theta=0$ means a purely public education system and $\theta=1$ means a purely private education system. This is a standard assumption in the literature (e.g., Glomm and Ravikumar 1992, Bénabou 1996, Fernandez and Rogerson 1998, and Takii and Tanaka 2009). When $\theta \in\{0,1\}$, public education differs from private education only in its financial system: the costs of education are financed by tuition in the private education system and by taxes in the public education system. Hence, 
we can ignore technical differences that have nothing to do with the definition of public education and private education.

The utility maximization problem of the young individual in household $i$ is characterized as follows. The young individual of household $i$ chooses consumption when young $c_{t}^{y, i}$, his/her child's private education expenditure $e_{t}^{i}$ and consumption when old $c_{t+1}^{o, i}$ to maximize his/her utility:

$$
\ln c_{t}^{y, i}+\beta E\left(\ln h_{t+1}^{i}\right)+\gamma \ln c_{t+1}^{o, i}
$$

subject to the budget constraint:

$$
c_{t}^{y, i}+e_{t}^{i}+b_{t}^{i}=\left(1-\tau_{t}\right) w_{t}\left(h_{t}^{i}\right), c_{t+1}^{o, i}=R_{t+1} b_{t}^{i}
$$

and the production function of human capital (5), where $R_{t+1}$ is the gross market interest rate and $E(\cdot)$ denotes the expectation operator.

From this utility maximization problem, we have the optimal private education and savings for the young individual $i$ at date $t$ :

$$
\begin{aligned}
e_{t}^{i} & =\lambda(\theta)\left(1-\tau_{t}\right) w_{t}\left(h_{t}^{i}\right), \\
b_{t}^{i} & =\frac{\gamma}{1+\gamma+\alpha \beta \theta}\left(1-\tau_{t}\right) w_{t}\left(h_{t}^{i}\right),
\end{aligned}
$$

where $\lambda(\theta)=\frac{\alpha \beta \theta}{1+\gamma+\alpha \beta \theta}$. Hence, each individual allocates constant fractions of his/her own disposable income to each current and future period's consumption, and the private education of his/her child only under the private education system.

Stock Market: Let $B_{t}$ denote total savings at date $t$, that is:

$$
B_{t}=\frac{\gamma}{1+\gamma+\alpha \beta \theta} L S_{t} Y_{t}
$$

The young individual invests all his/her savings in an index fund. The index consists of all stocks issued by all the firms. The total return from the index fund at date $t$ 
is equal to the total dividend plus the value of the index fund at date $t+1$. As the total savings must equal the value of the index fund at each period, the stock market clearing condition implies that:

$$
R_{t+1} B_{t}=B_{t+1}+\Pi_{t+1}
$$

where $\Pi_{t+1}=\left(1-L S_{t+1}\right) Y_{t+1}$ is the total profits earned by all jobs at date $t+1$, and $R_{t+1}$ is the gross rate of return from the index fund. This market clearing condition determines endogenously the gross rate of return $R_{t+1}$.

Choice of Tax Rate: Each young individual votes for a tax rate that maximizes his/her lifetime utility. ${ }^{7}$ Following Takii and Tanaka (2009), we assume that when voters choose their favorite tax rate, they take the future interest rate as given. ${ }^{8}$

\footnotetext{
${ }^{7}$ If the young individuals have the right to vote, then $\tau^{*}$ is chosen by unanimity. Even if both young and old individuals are enfranchised, $\tau_{t}=\tau^{*}$ is chosen by a majority because $\tau^{*}$ is always supported by at least half of the population (that is, all the young individuals always support $\left.\tau_{t}=\tau^{*}\right)$. Moreover, our model and the results are fully robust to the presence of positive population growth if job opportunities grow at the same rate and keep the same distribution. With positive population growth, the young are a strict majority, and thus $\tau_{t}=\tau^{*}$ is chosen in equilibrium even if the old are also enfranchised.

${ }^{8}$ In principle, the choice of tax rate affects the next period's interest rate through the aggregate variables. Here, we assume that voters ignore this effect of tax choice. Some may wonder whether this ignorance contradicts the assumption of rationality. However, to estimate the impact of tax on the interest rate, a household must calculate how other households in the current generation respond to the changes in the tax rate, how their responses change the distribution of human capital in the future generation, how the changes in the distribution of human capital influence the future tax rate that is voted on by future generations, and so on. These calculations allow a household to compute the changes not only in current aggregate savings, but also in future aggregate savings and future aggregate profits that determine the interest rate. We find that this process requires unreasonable rationality from agents and it is realistic to assume that agents take the future interest rate as given. Alternatively, we could assume a small open economy to derive our results without relying
} 
Hence, they vote for a tax rate that resolves the trade-off between the benefit from and the tax burden for public education.

The objective function (that is, indirect lifetime utility) in the tax choice problem of the young individual in family $i$ is given by:

$$
\begin{aligned}
& \ln \left[\left(1-\tau_{t}\right) w\left(h_{t}^{i}\right)_{t}-\lambda(\theta)\left(1-\tau_{t}\right) w_{t}\left(h_{t}^{i}\right)-\frac{\gamma}{1+\gamma+\alpha \beta \theta}\left(1-\tau_{t}\right) w_{t}\left(h_{t}^{i}\right)\right] \\
& +\beta E\left(\ln \left[\xi_{t+1}^{i}\left(\left(\lambda(\theta)\left(1-\tau_{t}\right) w_{t}\left(h_{t}^{i}\right)\right)^{\theta}\left(\tau_{t} \int w_{t}\left(h_{t}^{i}\right) d i\right)^{1-\theta}\right)^{\alpha}\right]\right) \\
& +\gamma \ln \left[R_{t+1} \frac{\gamma}{1+\gamma+\alpha \beta \theta}\left(1-\tau_{t}\right) w_{t}\left(h_{t}^{i}\right)\right]
\end{aligned}
$$

The favorite tax rate of the household with labor income $w_{t}\left(h_{t}^{i}\right)$ is given by:

$$
\tau_{t}^{*}=\frac{(1-\theta) \alpha \beta}{1+\gamma+\alpha \beta} \equiv \tau^{*}(\theta)
$$

Hence, $\tau^{*}(\theta)$ is chosen by unanimity.

\section{Public vs. Private Education System in the Short Run}

In this section, we derive the dynamics of GDP and examine how a change in the education system influences GDP dynamics. It is shown that an increase in $\sigma_{z}^{2}$ increases the benefits from private education.

Substituting equations (3), (6) and (7) into equation (5), we can derive the following dynamics of human capital of the dynasty $i$.

$$
\begin{aligned}
\ln h_{t+1}^{i}= & \alpha \theta\left(\frac{\sigma_{z}+v \sigma_{h, t}}{\sigma_{h, t}}\right)\left(\ln h_{t}^{i}-\mu_{h, t}\right)+\ln \xi_{t+1}^{i} \\
& +\alpha \ln L S_{t}+\alpha \theta\left[\mu_{z}+v \mu_{h, t}\right]+\alpha(1-\theta) \ln Y_{t}+C(\theta),
\end{aligned}
$$

on this controversial assumption. This alternative assumption makes the interest rate constant and therefore removes the effect of tax on the interest rate without changing our results. 
where $C(\theta)=\ln \left[\left(\lambda(\theta)\left(1-\tau^{*}(\theta)\right)\right)^{\alpha \theta}\left(\tau^{*}(\theta)\right)^{\alpha(1-\theta)}\right]$. Assume that ability is i.i.d. drawn from the log-normal distribution $\ln \xi_{t+1}^{i} \sim N\left(-\sigma_{a}^{2} / 2, \sigma_{a}^{2}\right)$. Because $\ln h_{t}^{i} \sim$ $N\left(\mu_{h, t}, \sigma_{h, t}^{2}\right)$, the human capital of the next period is also log-normally distributed: $\ln h_{t+1}^{i} \sim N\left(\mu_{h, t+1}, \sigma_{h, t+1}^{2}\right)$ where

$$
\mu_{h, t+1}=\alpha v \mu_{h, t}+\alpha \ln \left[\frac{v \sigma_{h, t}}{\sigma_{z}+v \sigma_{h, t}}\right]+\frac{\alpha(1-\theta)\left(\sigma_{z}+v \sigma_{h, t}\right)^{2}}{2}-\frac{\sigma_{a}^{2}}{2}+\alpha \mu_{z}+\ln C(\theta),
$$

and

$$
\sigma_{h, t+1}^{2}=\sigma_{a}^{2}+\alpha^{2} \theta^{2}\left(\sigma_{z}+v \sigma_{h, t}\right)^{2}
$$

These two equations fully describe the dynamics of the distribution of $\ln h_{t}$. Substituting equations (8) and (9) into equation (4), we can derive the dynamics of the logarithm of GDP and the standard deviation of log income as functions of the parameter of the education system $\theta$.

Proposition 1 The dynamics of $\ln Y_{t+1}$ and $\sigma_{w, t+1}$ can be given as functions of $\theta$ :

$$
\begin{aligned}
\ln Y_{t+1} & =\ln E(z)+\ln E\left[h_{t+1}^{v}\right]+\ln A S_{t+1} \\
\ln E\left[h_{t+1}^{v}\right] & =\alpha v \ln w_{t}^{e}+H C_{t}+C(\theta) \\
\ln w_{t}^{e} & =\ln L S_{t}+\ln Y_{t}
\end{aligned}
$$

and

$$
\sigma_{w, t+1}=\sigma_{z}+v \sigma_{h, t+1}=\sigma_{z}+v \sqrt{\sigma_{a}^{2}+\alpha^{2} \theta^{2} \sigma_{w, t}^{2}}
$$

where

$$
\begin{aligned}
\ln A S_{t+1} & =v \sigma_{z} \sigma_{h, t+1}=v \sigma_{z} \sqrt{\sigma_{a}^{2}+\alpha^{2} \theta^{2} \sigma_{w, t}^{2}} \\
\ln H C_{t} & =-v(1-v) \frac{\sigma_{a}^{2}}{2}-\alpha \theta v(1-\alpha \theta v) \frac{\sigma_{w, t}^{2}}{2} \\
\ln L S_{t} & =\ln \left(1-\frac{\sigma_{z}}{\sigma_{w, t}}\right) .
\end{aligned}
$$


Equation (10) shows that $Y_{t+1}$ can be decomposed into average productivity $E(z)$, aggregate human capital $E\left[h_{t+1}^{v}\right]$, and the productivity gains from assignment $A S_{t+1}$. Equation (11) shows that aggregate human capital is decomposed into the contribution of average labor income $w_{t}^{e}$, the reduction in aggregate human capital because of the heterogeneity of income and ability $H C_{t}$, and other constants $C(\theta)$. Finally, equation (12) shows that average labor income increases if either current GDP $Y_{t}$ or labor share $L S_{t}$ increases. These three equations describe the dynamics of $Y_{t}$ given the dynamics of $\sigma_{w, t}$. The dynamics of $\sigma_{w, t}$ are determined solely by equation (13), which shows that the variation of labor income depends not only on the variation of human capital, but also on that of job-specific productivity.

The component $H C_{t}$ summarizes a reduction in aggregate human capital because of the heterogeneity of income and ability. To guarantee the existence of a globally stable stationary distribution, it is shown later that we must assume $\alpha v \theta<1$. Given this assumption, equation (15) shows that a rise in $\sigma_{w, t}$ lowers the aggregate measure of human capital $E\left[h_{t+1}^{v}\right]$. This is the standard effect in previous studies: an increase in inequality lowers aggregate human capital.

The novel part of this paper is summarized by the component $A S_{t+1}$. It captures the productivity gains from assignment. As argued before, a rise in $\sigma_{h, t+1}$ increases this gain. Because a rise in $\sigma_{w, t}$ increases $\sigma_{h, t+1}$, it might be possible that a rise in inequality raises GDP in the next period.

Next, we identify the benefits and costs of an egalitarian education policy using the dynamics derived above. To do so, we compare a purely public education system $(\theta=0)$ with a purely private education system $(\theta=1)$, following the previous literature such as Glomm and Ravikumar (1992), Fernandez and Rogerson (1998), and Takii and Tanaka (2009).

Imagine an economy with the distribution of household income $\ln w_{t}\left(h_{t}^{i}\right) \sim N\left(\mu_{w, t}, \sigma_{w, t}^{2}\right)$. 
Suppose that the government of the economy is about to choose either the purely private $(\theta=1)$ or the purely public $(\theta=0)$ education system. Starting from this income distribution, the difference in the next period's GDP under these two systems is given by:

$$
\begin{aligned}
\ln Y_{t+1}^{\text {Pri }}-\ln Y_{t+1}^{\text {Pub }} & =\left[\ln A S_{t+1}^{\operatorname{Pr} i}-\ln A S_{t+1}^{\text {Pub }}\right]+\left[\ln H C_{t}^{\operatorname{Pr} i}-\ln H C_{t}^{\text {Pub }}\right] \\
& =v \sigma_{z}\left[\sqrt{\sigma_{a}^{2}+\alpha^{2} \sigma_{w, t}^{2}}-\sigma_{a}\right]-\frac{\alpha v(1-\alpha v) \sigma_{w, t}^{2}}{2}
\end{aligned}
$$

where $X_{t}^{P u b}$ and $X_{t}^{P r i}$ are $X_{t}$ under the public education system $(\theta=0)$ and the private education system $(\theta=1)$, respectively. Note that as $C(0)=C(1), C(\theta)$ is canceled out in equation (17).

The first term of this equation captures the productivity gains from assignment, and the second term captures human capital loss because of heterogeneous income. On the one hand, the productivity gains from assignment are always positive because $\sigma_{h, t+1}$ is larger under the private education system. On the other hand, the private education system always suffers from human capital loss, because income inequality reduces aggregate human capital in the next period. Whether or not the private education system increases GDP depends on the strength of these two opposite effects. The following proposition is immediate from equation (17).

Proposition 2 The relative advantages of the private education system depend on $\sigma_{z}$ in the following sense.

1. When $\sigma_{z}=0$, the public education system always generates larger GDP than the private system does.

2. A rise in $\sigma_{z}$ increases the relative advantages of the private education system:

$$
\frac{d\left(\ln Y_{t+1}^{P r i}-\ln Y_{t+1}^{P u b}\right)}{d \sigma_{z}}=v\left[\sqrt{\sigma_{a}^{2}+\alpha^{2} \sigma_{w, t}^{2}}-\sigma_{a}\right]>0 .
$$


3. The private education system attains higher GDP than the public system does if:

$$
\sigma_{z} \geq \frac{\alpha(1-\alpha v) \sigma_{w, t}^{2}}{2\left[\sqrt{\sigma_{a}^{2}+\alpha^{2} \sigma_{w, t}^{2}}-\sigma_{a}\right]} .
$$

Proposition 2 shows how the relative advantages of the private education system change as $\sigma_{z}$ becomes large. When $\sigma_{z}=0$, as there is no variation in jobs, there is no assignment problem. Hence, there are no productivity gains from assignment. In this case, the public education system always dominates the private education system. This is the standard result emphasized in previous studies.

When $\sigma_{z}>0$, differences in education systems can influence the pattern of assignment. This proposition shows that the advantage of the private education system increases as $\sigma_{z}$ increases. Note that a rise in $\sigma_{z}$ increases the marginal productivity of highly talented people. As the private education system can provide more talented people, a rise in $\sigma_{z}$ increases productivity gains from the private education system. This proposition also tells us that the productivity gains from assignment can outweigh the human capital loss if $\sigma_{z}$ is sufficiently large. Hence, if job opportunities have large variations, an education policy may face a serious efficiency-equity trade-off.

Compared with the impacts of $\sigma_{z}$, it is not clear how a rise in $\sigma_{w, t}^{2}$ influences the relative advantages of the private education system. On the one hand, it increases human capital loss because of a rise in inequality. It makes equalizing income and thus the public education system more beneficial. On the other hand, as a rise in $\sigma_{w, t}^{2}$ makes it likely to have more highly skilled workers, it also increases the productivity gains from assignment. Taking the derivative with respect to $\sigma_{w, t}^{2}$ in equation (17), it is shown that:

$$
\frac{d\left(\ln Y_{t+1}^{P r i}-\ln Y_{t+1}^{P u b}\right)}{d \sigma_{w, t}^{2}}>0, \text { iff } \sigma_{w, t}^{2}<\frac{\sigma_{z}^{2}}{(1-\alpha v)^{2}}-\frac{\sigma_{a}^{2}}{\alpha^{2}}
$$


If $\sigma_{w, t}^{2}$ is small enough, a rise in inequality increases the relative advantages of a private education system; if it is large enough, the opposite is true. The cutoff point depends on $\sigma_{z}^{2}$. Note that if $\sigma_{z}^{2}=0$, a rise in inequality always moves in favor of the public education system. When $\sigma_{z}^{2}=0$, there are no productivity gains from assignment. Hence, equalizing income is always beneficial. However, when $\sigma_{z}^{2}$ is large, the demand for highly skilled labor is large, which makes private education more beneficial.

This analysis suggests that certain results in this section may not be relevant for the long run. As a rise in $\sigma_{z}$ also increases $\sigma_{w, t+1}^{2}$, there are indirect effects that may outweigh the direct effect. This is the theme in the next section.

\section{Public vs. Private Education System in the Long Run}

In this section, we analyze how a rise in $\sigma_{z}$ influences the relative advantages of education systems when an aggregate economy reaches a steady state. The steadystate level of $\sigma_{w, t}^{2}$ is endogenously determined as a function of $\sigma_{z}$. Hence, it is not clear how a rise in $\sigma_{z}$ changes the impacts of changes in education systems on GDP in the long run. First, we show that an aggregate economy converges to a unique steady state. Later, we analyze how a rise in $\sigma_{z}$ changes the relative advantages of a private education system when the economy is in its steady state.

First, we prove that there is a globally stable stationary income distribution. Note that both $\ln Y_{t}$ and $\sigma_{w, t}$ converge to stationary points if and only if $\mu_{h, t}$ and $\sigma_{h, t}$ in equations (8) and (9) converge to stationary points. Assuming that $\alpha v<1$, it is easy to see that $\mu_{h, t}$ converges to a stationary point if $\sigma_{h, t}$ converges to a stationary point. Hence, by examining the convergence of $\sigma_{h, t}$, we can prove the following proposition. 
Its formal proof is in the appendix.

Proposition 3 Assume that $\alpha v \theta<1$. The economy converges monotonically to a unique and globally stable stationary income distribution.

When the economy reaches the stationary distribution, $\sigma_{w, t}$, which affects GDP, is endogenously determined. The next proposition summarizes the dependent relationship of GDP on the exogenous parameters.

Proposition 4 The logarithm of the steady-state GDP is expressed by:

$$
\ln Y^{*}=\frac{1}{1-\alpha v}\left[\ln E(z)+\alpha v \ln L S^{*}+\ln H C^{*}+\ln A S^{*}+C(\theta)\right]
$$

where

$$
\begin{aligned}
\ln A S^{*} & =v \sigma_{z} \sqrt{\sigma_{a}^{2}+\alpha^{2} \theta^{2}\left(\sigma_{w}^{*}\right)^{2}} \\
\ln H C^{*} & =-v(1-v) \frac{\sigma_{a}^{2}}{2}-\alpha \theta v(1-\alpha \theta v) \frac{\left(\sigma_{w}^{*}\right)^{2}}{2} \\
\ln L S^{*} & =\ln \left(1-\frac{\sigma_{z}}{\sigma_{w}^{*}}\right)
\end{aligned}
$$

and

$$
\sigma_{w}^{*}=\frac{\sigma_{z}+\sqrt{\alpha^{2} \theta^{2} v^{2} \sigma_{z}^{2}+\left(1-\alpha^{2} v^{2} \theta^{2}\right) v^{2} \sigma_{a}^{2}}}{1-\alpha^{2} v^{2} \theta^{2}} .
$$

Similarly to the previous section, $\theta$ influences GDP through the productivity gains from assignment $A S^{*}$, a reduction in aggregate human capital because of the heterogeneity of income and ability $H C^{*}$, and other constants $C(\theta)$.

However, a change in $\theta$ has an additional indirect effect. As it changes $\sigma_{w}^{*}$, it also changes the labor share $L S^{*}$. A rise in the variation of labor income $\sigma_{w}^{*}$ increases the variation of skill at the stationary point $\sigma_{h}^{*}$, which increases the rent from skill. To understand the logic behind this argument, imagine what would happen if $\sigma_{h}^{*}=0$. In this case, everybody has the same skill, and thus a firm can always find alternative 
workers. Therefore, the wage payments for all workers are at the reservation wage, which is 0 by assumption, and a firm takes all the surplus. This mechanism is equivalent to that of a Ricardian rent model. When workers have more variation in skill, skilled workers receive more than the reservation wage because it is difficult to find alternative workers. As the reservation wage does not change, unskilled workers are still guaranteed to receive the reservation wages. This mechanism increases the labor share. As an increase in the labor share increases the average labor income, it raises the average amount of investment in human capital and, therefore, GDP.

Similarly to the previous section, we compare the productive impact of the purely private education system $(\theta=1)$ and the purely public education system $(\theta=0)$ :

$$
\begin{aligned}
& \ln \left(Y^{*}\right)^{\operatorname{Pr} i}-\ln \left(Y^{*}\right)^{P u b} \\
& =\frac{\left\{\begin{array}{c}
\alpha v\left[\ln \left(L S^{*}\right)^{\operatorname{Pr} i}-\ln \left(L S^{*}\right)^{P u b}\right]+\left[\ln \left(A S^{*}\right)^{\operatorname{Pr} i}-\ln \left(A S^{*}\right)^{P u b}\right] \\
+\left[\ln \left(H C^{*}\right)^{\operatorname{Pr} i}-\ln \left(H C^{*}\right)^{P u b}\right]
\end{array}\right\}}{1-\alpha v},
\end{aligned}
$$

where

$$
\begin{aligned}
& \ln \left(L S^{*}\right)^{\operatorname{Pr} i}-\ln \left(L S^{*}\right)^{P u b}=\ln \left(1-\frac{\sigma_{z}}{\left(\sigma_{w}^{*}\right)^{\operatorname{Pr} i}}\right)-\ln \left(\frac{v \sigma_{a}}{\sigma_{z}+v \sigma_{a}}\right), \\
& \ln \left(A S^{*}\right)^{\operatorname{Pr} i}-\ln \left(A S^{*}\right)^{P u b}=\sigma_{z}\left[\sqrt{\left(v \sigma_{a}\right)^{2}+(\alpha v)^{2}\left[\left(\sigma_{w}^{*}\right)^{\operatorname{Pr} i}\right]^{2}}-v \sigma_{a}\right], \\
& \ln \left(H C^{*}\right)^{\operatorname{Pr} i}-\ln \left(H C^{*}\right)^{P u b}=-\frac{\alpha v(1-\alpha v)\left[\left(\sigma_{w}^{*}\right)^{\text {Pri }}\right]^{2}}{2}, \\
& \left(\sigma_{w}^{*}\right)^{\operatorname{Pr} i}=\frac{\sigma_{z}+\sqrt{(\alpha v)^{2} \sigma_{z}^{2}+\left[\left(1-(\alpha v)^{2}\right]\left(v \sigma_{a}\right)^{2}\right.}}{1-(\alpha v)^{2}} .
\end{aligned}
$$

Note that $C(\theta)$ is canceled out in equation (18) for the same reason as in the previous section.

Because a rise in $\sigma_{z}$ increases $\left(\sigma_{w}^{*}\right)^{\operatorname{Pr} i}$, not only the productivity gains from assignment $\ln \left(A S^{*}\right)^{\operatorname{Pr} i}-\ln \left(A S^{*}\right)^{P u b}$ but also human capital loss because of the variation of 
income $\left|\ln \left(H C^{*}\right)^{\operatorname{Pr} i}-\ln \left(H C^{*}\right)^{P u b}\right|$ increases. In addition, $\ln \left(L S^{*}\right)^{\operatorname{Pr} i}-\ln \left(L S^{*}\right)^{P u b}$ is also influenced by $\sigma_{z}$ and $\left(\sigma_{w}^{*}\right)^{\operatorname{Pr} i}$. These observations imply that $\frac{d\left(\ln Y^{\operatorname{Pr} i}-\ln Y^{P u b}\right)}{d \sigma_{z}}$ can be positive or negative, and thus there is no monotonic relationship. However, we can provide the following weaker statement about the relationship between the relative advantage of the public education system and the variations in jobs as in Proposition 5 (the proof is in the appendix).

Proposition 5 There exist $\underline{\sigma_{z}}$ and $\overline{\sigma_{z}}\left(0<\underline{\sigma_{z}} \leq \overline{\sigma_{z}}<\infty\right)$ such that: (i) if $\sigma_{z}<\underline{\sigma_{z}}$, $\ln Y^{P u b}>\ln Y^{\operatorname{Pr} i} ;$ (ii) if $\bar{\sigma}_{z}<\sigma_{z}, \ln Y^{P u b}<\ln Y^{\operatorname{Pr} i}$.

This proposition says that if $\sigma_{z}$ is large enough (small enough), productivity gains from the private education system (the public education system) are larger. Therefore, this proposition confirms the main messages of this paper: if job opportunities have large variations, an education policy may face a serious efficiency-equity tradeoff.

\section{Concluding Remarks}

This paper compared private and public education systems when the labor market is characterized by positive assortative matching between the productivity of jobs and skill. It pointed out that there is a neglected role for an education policy that cannot be captured by a measure of aggregate human capital. More specifically, it showed that private education can increase the productivity gains from assignment by increasing the availability of highly educated people, and that if job opportunities have large variations in productivity, an education policy may face a serious efficiencyequity trade-off.

The results of the model must be interpreted with caution. We have no intention of claiming that a private education system always increases gains from assignment. 
Of course, it depends on the structure of the production side. We selected Sattinger's (1979) assignment model as our production structure because it clarifies how an education policy influences gains from assignment by changing the distribution of human capital and how the novel mechanism changes the results in previous studies. It is possible to develop a production structure for which a public education system can improve gains from assignment. In the rest of paper, we speculated about alternative specifications of the production structure to examine the robustness of our results.

First, we presumed that productivity differs across jobs because there exist jobspecific resources. This creates Ricardian rent, which can be shared with assigned workers. However, the advantage of a job might be the result of other factors such as unnecessary regulation. If this is the case, skilled workers may be misallocated to an unsuitable position, which may lower productivity. This reasoning suggests that the relative advantages of a private education system may be lower when a market is heavily distorted.

Second, we assumed that jobs are perfect substitutes in a firm . In fact, our assumption of perfectly substitutable jobs is not as restrictive as it looks. Alternatively, we could assume that a firm has the following production function and maximizes its profits:

$$
y(z)=z\left[\int_{0}^{1}\left(h_{t}^{i}\right)^{\psi} d i\right]^{\frac{v}{\psi}}, \psi<1, v>0 .
$$

Workers are complementary to each other, but this production function derives exactly the same results as those in this paper. In this case, a firm employs the same quality of workers and segregation occurs across firms. The assumption of perfectly substitutable jobs is made only for simplicity.

Of course, this does not mean that complementarity is unimportant. As emphasized by Bénabou (1996) and Takii and Tanaka (2009), if the firm faces a mo- 
nopolistically competitive market, the complementarity among goods favors a more homogeneous skill distribution. Hence, it is likely that the complementarity matters in this context.

These arguments suggest that if we wish to identify from data how an education policy influences the gains from assignment, we need to be careful not only about the heterogeneity across jobs, but also about the source of the heterogeneities and how heterogeneous factors interact. Incorporating these aspects into the model and quantitatively assessing an education policy would be interesting future research.

\section{A Appendix}

Proof of Proposition 3: As $\alpha v<1$ is guaranteed by the assumption, it is obvious that equation (8) converges to the steady state if equation (9) converges to the steady state. Hence, we only prove the convergence of equation (9). Define:

$$
\sigma_{h, t+1}=\left[\sigma_{a}^{2}+\alpha^{2} \theta^{2}\left(\sigma_{z}+v \sigma_{h, t}\right)^{2}\right]^{1 / 2} \equiv f\left(\sigma_{h, t}\right)
$$

Because $f\left(\sigma_{h, t}\right)$ is continuous in $\sigma_{h, t}$ and $f(0)>0$, it is sufficient for the existence of a steady state to show that $\lim _{\sigma_{h, t} \rightarrow \infty} f^{\prime}\left(\sigma_{h, t}\right)<1$. In fact:

$$
\lim _{\sigma_{h, t} \rightarrow \infty} f^{\prime}\left(\sigma_{h, t}\right)=\lim _{\sigma_{h, t \rightarrow \infty}} \alpha v \theta \sqrt{\frac{\alpha^{2} \theta^{2}\left(\sigma_{z}+v \sigma_{h, t}\right)^{2}}{\sigma_{a}^{2}+\alpha^{2} \theta^{2}\left(\sigma_{z}+v \sigma_{h, t}\right)^{2}}}=\alpha v \theta<1 .
$$

Hence there exists at least one steady state $\sigma_{h, t}$ (and thus a steady-state distribution of income). For the uniqueness and stability of the steady state, note that:

$$
f^{\prime}\left(\sigma_{h, t}\right)=\alpha v \theta \sqrt{\frac{\alpha^{2} \theta^{2}\left(\sigma_{z}+v \sigma_{h, t}\right)^{2}}{\sigma_{a}^{2}+\alpha^{2} \theta^{2}\left(\sigma_{z}+v \sigma_{h, t}\right)^{2}}} \in[0,1] .
$$

Proof of Proposition 5: Note that when $\sigma_{z}=0, \ln Y^{P r i}-\ln Y^{P u b}<0$. Because $\ln Y^{P r i}-\ln Y^{P u b}$ is continuous in $\sigma_{z}$, there must exist $\underline{\sigma_{z}}>0$ such that $\ln Y^{\text {Pri }}-$ 
$\ln Y^{P u b}<0$ for any $\sigma_{z} \in\left[0, \underline{\sigma_{z}}\right)$. Hence, it is enough to show that there exists $\bar{\sigma}_{z}<\infty$ such that $\ln Y^{P r i}-\ln Y^{P u b}>0$ for any $\sigma_{z} \in\left(\bar{\sigma}_{z}, \infty\right)$.

For the first term in equation (18), note that $\alpha v\left[\ln \left(L S^{*}\right)^{P r i}-\ln \left(L S^{*}\right)^{P u b}\right]=$ $\alpha v\left[\ln \left(1-\frac{\sigma_{z}}{\left(\sigma_{w}^{*}\right)^{P r i}}\right)-\ln \left(\frac{v \sigma_{a}}{\sigma_{z}+v \sigma_{a}}\right)\right]>0$. This is because the steady-state standard deviation of income under the private system is always larger than that under the public system, and because the labor share is increasing in the standard deviation of income. This observation implies that $\ln Y^{P r i}-\ln Y^{P u b}>0$ if the sum of the second and the third terms in equation (18) is nonnegative: $\left[\ln \left(A S^{*}\right)^{P r i}-\ln \left(A S^{*}\right)^{P u b}\right]+$ $\left[\ln \left(H C^{*}\right)^{\text {Pri }}-\ln \left(H C^{*}\right)^{P u b}\right] \geq 0$.

Next, note that $\left[\ln \left(A S^{*}\right)^{P r i}-\ln \left(A S^{*}\right)^{P u b}\right]+\left[\ln \left(H C^{*}\right)^{P r i}-\ln \left(H C^{*}\right)^{P u b}\right] \geq 0$ if and only if

$$
\left[\left(\sigma_{w}^{*}\right)^{P r i}\right]^{2} \leq \bar{\sigma}_{w} \equiv \frac{4 \sigma_{z}}{1-\alpha v}\left[\frac{\sigma_{z}}{1-\alpha v}-\frac{\sigma_{a}}{\alpha}\right]
$$

This observation comes from direct calculations. It implies that for a given $\sigma_{z}$, if $\left[\left(\sigma_{w}^{*}\right)^{P r i}\right]^{2} \leq \bar{\sigma}_{w}$, then $\ln Y^{P r i}-\ln Y^{P u b}>0$. Hence, defining $\widetilde{\sigma}_{z} \equiv \frac{\sigma_{z}}{\sigma_{a}}$, we must show that there exists a unique $\overline{\widetilde{\sigma}}_{z} \in\left(\frac{1-\alpha v}{\alpha}, \infty\right)$ such that $\left[\left(\sigma_{w}^{*}\right)^{\operatorname{Pr} i}\right]^{2} \leq \bar{\sigma}_{w}$ for any $\widetilde{\sigma}_{z} \geq \overline{\widetilde{\sigma}}_{z}$. Then, for a given $\sigma_{a}>0$, the threshold level of $\sigma_{z}\left(\bar{\sigma}_{z}\right.$ in the proposition) is uniquely given by $\bar{\sigma}_{z}=\left(\overline{\widetilde{\sigma}}_{z}\right) \sigma_{a}$.

Consider the following function of $\widetilde{\sigma}_{z} \in\left(\frac{1-\alpha v}{\alpha}, \infty\right)$ :

$$
g\left(\widetilde{\sigma}_{z}\right)=\frac{\left(\frac{1+v \sqrt{\alpha^{2}+\left(1-\alpha^{2} v^{2}\right) \frac{1}{\left(\widetilde{\sigma}_{z}\right)^{2}}}}{1-\alpha^{2} v^{2}}\right)^{2}}{\frac{4}{1-\alpha v}\left[\frac{1}{1-\alpha v}-\frac{1}{\alpha \widetilde{\sigma}_{z}}\right]} .
$$

Observe that $g\left(\widetilde{\sigma}_{z}\right)=\frac{\left[\left(\sigma_{w}^{*}\right)^{P r i}\right]^{2}}{\bar{\sigma}_{w}^{2}}$ for $\widetilde{\sigma}_{z} \in\left(\frac{1-\alpha v}{\alpha}, \infty\right)$. Hence, it is enough to show there 
exists a unique $\overline{\widetilde{\sigma}}_{z} \in\left(\frac{1-\alpha v}{\alpha}, \infty\right)$ such that for any $\widetilde{\sigma}_{z}>\overline{\widetilde{\sigma}}_{z}, g\left(\widetilde{\sigma}_{z}\right)<1$. Note that

$$
\begin{aligned}
\lim _{\widetilde{\sigma}_{z} \rightarrow \infty} g\left(\widetilde{\sigma}_{z}\right) & =\frac{\left(\frac{1+v \sqrt{\alpha^{2}}}{1-\alpha^{2} v^{2}}\right)^{2}}{\frac{4}{1-\alpha v}\left[\frac{1}{1-\alpha v}\right]}=\frac{\left(\frac{1+\alpha v}{(1-\alpha v)(1+\alpha v)}\right)^{2}}{\frac{4}{(1-\alpha v)^{2}}} \\
& =\frac{\frac{(1+\alpha v)^{2}}{(1-\alpha v)^{2}(1+\alpha v)^{2}}}{\frac{4}{(1-\alpha v)^{2}}}=\frac{1}{4}<1,
\end{aligned}
$$

and $\lim _{\widetilde{\sigma}_{z} \rightarrow \frac{1-\alpha v}{\alpha}+0} g\left(\widetilde{\sigma}_{z}\right)=\infty$. Because $g\left(\widetilde{\sigma}_{z}\right)$ is continuous and strictly decreasing in $\widetilde{\sigma}_{z} \in\left(\frac{1-\alpha v}{\alpha}, \infty\right)$,there exists a unique threshold level of $\widetilde{\sigma}_{z}, \overline{\widetilde{\sigma}}_{z} \in\left(\frac{1-\alpha v}{\alpha}, \infty\right)$ such that $g\left(\widetilde{\sigma}_{z}\right)<1$ for any $\widetilde{\sigma}_{z}>\overline{\widetilde{\sigma}}_{z}$. This completes the proof.

\section{References}

[1] Abowd M., John, Francis Kramarz, and David N. Margolis (1999), "High Wage Workers and High Wage Firms," Econometrica, Vol. 67, No. 2, March, 251-333.

[2] Acemoglu, Daron (1997), "Matching, Heterogeneity and the Evolution of Income Distribution," Journal of Economic Growth, Vol. 1, 66-92.

[3] Baily, Martin, Neil C. Hulten and David Campbell (1992), "Productivity Dynamics in Manufacturing Plants," Brookings Papers on Economic Activity. Microeconomics, 187-249.

[4] Becker, Gary S. (1973), "A Theory of Marriage: Part 1," Journal of Political Economy, 81, 813-846.

[5] Bénabou, Roland. (1996), "Heterogeneity, Stratification, and Growth: Macroeconomic Implications of Community Structure and School Finance," American Economic Review, Vol. 86, 3, 584-609. 
[6] Bénabou, Roland. (2002), "Tax and Education Policy in a Heterogeneous-Agent Economy: What Levels of Redistribution Maximize Growth and Efficiency?" Econometrica, 70 (2), 481-517.

[7] Bresnahan, Timothy F., Erik Brynjolfsson, and Lorin M. Hitt (2002), "Information Technology, Workplace Organization, and the Demand for Skilled Labor: Firm-Level Evidence," Quarterly Journal of Economics, Vol. 117, 339-376.

[8] Chandler, A. (1977), The Visible Hand: The Managerial Revolution in American Business, The Belknap Press of Harvard University Press, Cambridge, Massachusetts.

[9] Eeckhout, Jan and Philipp Kircher (2009), "Identifying Sorting - In Theory," PIER Working Paper, 09-007.

[10] Fernández, Raquel and Richard Rogerson (1998), "Public Education and the Dynamics of Income Distribution: A Quantitative Evaluation of Education Finance Reform," American Economic Review, Vol. 88, 4, 813-833.

[11] Glomm, Gerhard and B. Ravikumar (1992), "Public versus Private Investment in Human Capital: Endogenous Growth and Income Inequality," Journal of Political Economy, Vol. 100, 4, 818-834.

[12] Jovanovic, Boyan (1998), "Vintage Capital and Inequality," Review of Economic Dynamics, 1, 497-530.

[13] Jovanovic, Boyan (2009), "The Technology Cycle and Inequality," Review of Economic Studies, 76, 707-729.

[14] Katz, Lawrence F., and Kevin M. Murphy (1992), "Changes in Relative Wages, 1963-1987: Supply and Demand Factors, " Quarterly Journal of Economics, Vol. 107, February, 35-78. 
[15] Kremer, Michael. (1993), "The O-Ring Theory of Economic Development," Quarterly Journal of Economics, Vol. 108, August, 551-575.

[16] Krusell, Per, Lee E. Ohanian, José-Víctor Ohanian, Ríos-Rull, and Giovanni L. Violante (2000), "Capital-Skill Complementarity and Inequality: A Macroeconomic Analysis, " Econometrica, Vol. 68, No. 5 (September), 1029-1053.

[17] Loury, Glenn (1981), "Intergenerational Transfers and the Distribution of Earnings," Econometrica, 49, 843-867.

[18] Lopes de Melo, Rafael (2008), "Sorting in the Labor Market: Theory and Measurement," Mimeo.

[19] Mendes, Rute, Gerard van den Berg, and Maarten Lindeboom (2007), "An Empirical Assessment of Assortative Matching in the Labor Market," IZA Discussion Paper, No. 3053.

[20] Sattinger, Michael (1979), "Differential Rents and the Distribution of Earnings," Oxford Economic Papers, Vol. 31, March, 60-71.

[21] Sattinger, Michael (1980), Capital and the Distribution of Labor Earnings, North-Holland, Amsterdam.

[22] Takii, Katsuya (2008), "The Persistence of Differences in Productivity, Wages, Skill Mixes and Profits Between Firms in a Rapidly Changing Environment," OSIPP Discussion Paper: DP-2008-E-003, Osaka University, February.

[23] Takii, Katsuya and Ryuichi Tanaka (2009), "Does the Diversity of Human Capital Increase GDP?: A Comparison of Education Systems," Journal of Public Economics, Vol.93, No. 7-8, August, 998-1007. 